\title{
Bloqueio motor e sensitivo da lidocaína ou da ropivacaína peridural em cães
}

[Motor and sensitive blockade provided by epidural lidocaine or ropivacaine in dogs]

L.D. Thomas ${ }^{1}$, L.A.T. Francini ${ }^{2}$, J.F. Sinotti ${ }^{1}$, W.S. Chan ${ }^{3}$, J.T. Druziani ${ }^{2}$, F.B. Fukushima ${ }^{2}$

${ }^{1}$ Aluna de pós-graduação - Universidade Federal do Paraná - Palotina, PR

${ }^{2}$ Universidade Federal do Paraná - Palotina, PR

${ }^{3}$ Aluno de pós-graduação - Universidade Federal de Minas Gerais - Belo Horizonte, MG

\section{RESUMO}

Objetivou-se avaliar o bloqueio sensitivo e motor da administração peridural de $0,2 \mathrm{~mL} / \mathrm{kg}$ de duas concentrações de ropivacaína em comparação à lidocaína em cães. Utilizaram-se 24 cães, distribuídos em quatro grupos: $\mathrm{NaCl}$ a $0,9 \%$ (GS), lidocaína a $2 \%$ (GL), ropivacaína a $0,5 \%$ (GR5) e ropivacaína a $0,75 \%$ (GR7,5). Avaliaram-se a presença de movimentação espontânea, deambulação, sensibilidade superficial e profunda nos momentos cinco, 10, 15, 20, 25, 30, 45, 60, 90, 120, 180, 240 e 300 minutos após peridural. O retorno à movimentação espontânea foi semelhante entre GL $(42,50 \pm 6,12)$ e GR7,5 $(69,2 \pm 58,9)$. O tempo para deambulação foi mais prolongado em GR7,5 $(107,5 \pm 79,3)$ que em GS $(9,2 \pm 3,8)$ e em GR5 $(32,5 \pm 20,9)$. O retorno da sensibilidade profunda foi maior em GR 7,5 (152,5 $\pm 89,2)$ que em GS $(5,8 \pm$ $2,0)$, GR5 $(46,7 \pm 46,3)$ e GL $(52,5 \pm 20,7)$. O tempo de retorno da sensibilidade superficial foi maior em GR7,5 (205,0 \pm 129,3) que em GS (7,5 $\pm 2,7)$, GL (72,5 $\pm 19,9)$ e GR5 $(97,5 \pm 55,1)$. Apesar do retorno precoce da movimentação, ropivacaína $0,75 \%$ está relacionada a tempo prolongado de recuperação da função muscular e bloqueio sensitivo mais prolongado que lidocaína e ropivacaína $0,5 \%$.

Palavras-chave: anestésico local, bloqueio motor, bloqueio sensitivo, epidural

\section{ABSTRACT}

The aim of the present study was to evaluate the sensory and motor blockade of epidural $0.5 \%$ and $0.75 \%$ Ropivacaine or Lidocaine in dogs. Twenty-four dogs were distributed in four groups: $0.9 \% \mathrm{NaCl}(\mathrm{GS}), 2 \%$ lidocaine (GL), 0.5\% ropivacaine (GR5) and $0.75 \%$ ropivacaine (GR7.5). Spontaneous movement, ability to walk, superficial, and deep pain response were assessed 5, 10, 15, 20, 25, 30, 45, 60, 90, 120, 180, 240 and 300 minutes after epidural. Time to return to spontaneous movement was similar between GL (42.50 $\pm 6.12)$ and GR7.5 (69.2 \pm 58.9$)$. Time to return to ambulation was longer in GR7.5 (107.5 \pm 79.3$)$ than in $G S(9.2 \pm 3.8)$ and GR5 (32.5 \pm 20.9$)$. Time to recover deep sensitivity was longer in GR $7.5(152.5 \pm 89.2)$ than in $G S(5.8 \pm 2.0)$, GR5 (46.7 \pm 46.3$)$ and $G L(52.5 \pm 20.7)$. Time to return superficial sensitivity was longer in GR7.5 (205.0 \pm 129.3$)$ when compared to GS (7.5 \pm 2.7$)$, GL (72.5 \pm 19.9$)$ and GR5 (97.5 \pm 55.1$)$. Despite the early return of spontaneous movement, $0.75 \%$ ropivacaine is related to longer periods for muscle function recovery and longer sensory block than lidocaine and $0.5 \%$ ropivacaine.

Keywords: local anesthetic, motor block, sensitive block, epidural

\section{INTRODUÇÃO}

A anestesia peridural lombossacra (L7-S1) é uma técnica amplamente empregada para procedimentos cirúrgicos na medicina veterinária. Apesar de ser possível a administração de diferentes fármacos por essa via, o fármaco ideal deve promover analgesia prolongada, com mínimo bloqueio motor e ausência de efeitos sistêmicos adversos (Valverde, 2008). Dessa forma, a escolha do fármaco deve ser baseada na potência, na latência, na duração do efeito e no tipo de procedimento cirúrgico, levando em conta os possíveis efeitos adversos (Steagall et al., 2017). Entre os fármacos utilizados por via peridural, os anestésicos locais são os mais empregados. O mecanismo de ação dos anestésicos locais administrados pela via peridural envolve o bloqueio de canais para sódio

Recebido em30 de setembro de 2019

Aceito em 10 de abril de 2020

E-mail: lettyciadt@gmail.com 
presentes na membrana das células neuronais, e o tempo de ação é dependente da solubilidade lipídica e da afinidade com o receptor (GarciaPereira, 2018). As raízes espinhais são bloqueadas à medida que o anestésico local progride no espaço peridural, localizado entre a dura-máter e os limites do canal vertebral (Campoy et al., 2015). Sabe-se que o bloqueio sensitivo diferencial está associado não somente à ausência/presença de mielina ou ao tamanho da fibra nervosa a ser bloqueada, mas à capacidade do agente anestésico em promover bloqueio seletivo de fibras nociceptivas (fibras $\mathrm{C}$ e $\mathrm{A} \delta$ ) em relação a fibras motoras (fibas $\mathrm{A} \alpha$ ) (MartinFlores, 2013) .

A lidocaína é indicada para cirurgias com duração máxima de 120 minutos, embora o tempo de analgesia possa ser prolongado quando associada a opioides ou vasoconstritores (Almeida et al., 2010). A ropivacaína é amplamente utilizada na medicina humana e apresenta menor tempo de bloqueio motor quando comparada à bupivacaína; por possuir alta afinidade de ligação proteica, ao contrário da lidocaína, apresenta tempo de duração mais prolongado, de três a seis horas (Feldman et al., 1996; Jones, 2001; Adami et al., 2012). Para garantir o bloqueio peridural, um volume mínimo do agente anestésico é necessário, sem influência aparente da concentração no grau de bloqueio nervoso. Contudo, quando o volume mínimo é atingido, concentrações mais altas de anestésico podem aumentar a eficácia e a duração do efeito analgésico (Otero e Campoy, 2013).

Apesar de os mecanismos de ação desses fármacos já serem bem definidos, não há na literatura informações sobre o tempo exato de ação do bloqueio sensitivo e motor de diferentes concentrações de ropivacaína. Dessa forma, o objetivo do presente estudo foi avaliar o efeito da lidocaína e de duas concentrações de ropivacaína administradas por via peridural, em cães, sobre parâmetros sensitivos e motores.

\section{MATERIAL E MÉTODOS}

O estudo foi aprovado pelo Comitê de Ética e Uso de Animais da Universidade Federal do Paraná, Setor Palotina (Protocolo Ceua Palotina $\mathrm{n}^{\circ}$ 19/2015), e todos os tutores assinaram termo de consentimento livre e esclarecido. Foram utilizados 24 cães, machos, sem raça definida, não castrados, com peso corporal de $4 \mathrm{~kg}$ a $15 \mathrm{~kg}$, idade de nove meses a oito anos, classificados como
ASA I e II, com base em avaliação física e laboratorial que incluíram hemograma e dosagem sérica de ureia, creatinina, albumina, alanina aminotransferase e fosfatase alcalina.

Antes do ensaio, todos os cães foram submetidos a jejum alimentar de 12 horas e venóclise da veia cefálica cranial. Na sequência, procedeu-se à indução anestésica com fentanila $(3 \mu \mathrm{g} / \mathrm{kg}$, IV) e propofol $1 \%$, IV, dose-efeito, até perda de reflexos protetores e relaxamento mandibular. A anestesia geral foi mantida com isoflurano em vaporizador universal e sistema circular com reinalação parcial de gases. Os animais foram posicionados em decúbito esternal, com os membros pélvicos tracionados cranialmente. Em seguida, procedeu-se à peridural lombossacra (L7-S1), sendo a confirmação do correto posicionamento da agulha realizado pelo teste da gota pendente e pela ausência de deformação da bolha no anestésico. Os animais foram distribuídos aleatoriamente em quatro grupos: GS $(0,2 \mathrm{~mL} / \mathrm{kg}$ de solução $\mathrm{NaCl} 0,9 \%, \mathrm{n}=6)$, GL $(0,2 \mathrm{~mL} / \mathrm{kg}$ de lidocaína $2 \%$ sem vasoconstritor, $\mathrm{n}=6)$, GR5 $(0,2 \mathrm{~mL} / \mathrm{kg}$ de ropivacaína $5,0 \mathrm{mg} / \mathrm{mL}$ sem vasoconstritor, $\mathrm{n}=6)$, e GR7,5 $(0,2 \mathrm{~mL} / \mathrm{kg}$ de ropivacaína $7,5 \mathrm{mg} / \mathrm{mL}$ sem vasoconstritor, $\mathrm{n}=6$ ). A deposição do fármaco anestésico foi realizada durante dois minutos. A administração dos fármacos e as avaliações foram executadas pelo mesmo anestesista, sem conhecimento prévio do grupo a que os animais pertenciam.

Após a administração do tratamento, os animais foram posicionados em decúbito ventral, o fornecimento do anestésico foi interrompido e os pacientes foram acompanhados até a extubação, na presença de reflexo de deglutição. Os animais foram monitorados quanto aos seguintes parâmetros: capacidade de movimentação espontânea dos membros, caracterizada pela observação de qualquer movimento dos membros pélvicos na ausência de estimulação; capacidade de deambulação, caracterizada pelo retorno da capacidade de caminhar; presença de propriocepção em membros pélvicos bilateral; escore de atividade motora (Abelson et al., 2011) (Tab. 1) e sensibilidade superficial e profunda em membros pélvicos bilateral. A avaliação da sensibilidade foi realizada pelo pinçamento interdigital (sensibilidade superficial) e digital (sensibilidade profunda), executada sempre pelo mesmo avaliador, com pinça hemostática emborrachada,fechada até a primeira cremalheira. 
Tabela 1. Escala descritiva simples da atividade motora para avaliação de cães submetidos à administração de fármacos peridural

\begin{tabular}{cl}
\hline Escore & \multicolumn{1}{c}{ Descrição da atividade motora } \\
\hline 1 & $\begin{array}{l}\text { Sem fraqueza motora; marcha normal; capaz de ficar em pé e andar sem suporte } \\
\text { Fraqueza motora leve; anormalidade da marcha leve; capaz de ficar em pé e andar sem } \\
\text { suporte }\end{array}$ \\
3 & $\begin{array}{l}\text { Fraqueza motora moderada; requer assistência para ficar em pé e, então, é capaz de andar } \\
\text { com marcha anormal, porém sem assistência }\end{array}$ \\
4 & $\begin{array}{l}\text { Fraqueza motora acentuada; incapaz de ficar em pé ou andar sem ajuda } \\
5\end{array}$ \\
Paralisia; sem função motora voluntária evidente
\end{tabular}
Adaptado de Abelson et al. (2011).

A avaliação dos parâmetros descritos ocorreram aos cinco (T5), 10 (T10), 15 (T15), 20 (T20), 25 (T25), 30 (T30), 45 (T45), 60 (T60), 90 (T90), 120 (T120), 180 (T180), 240 (T240) e 300 (T300) minutos após a peridural. Os dados foram submetidos ao teste de normalidade KolmogorovSmirnov. Os dados paramétricos foram submetidos à ANOVA e à comparação entre grupos e entre tempos pelo teste Student Newman-Keuls. Os dados não paramétricos entre grupos no tempo foram submetidos à comparação pelo teste de Kruskal-Wallis e pos-hoc de Dunn. Para todas as comparações, considerou-se nível de significância de $5 \%$.

\section{RESULTADOS E DISCUSSÃO}

A média geral de peso dos animais foi de 7,72 \pm $3,16 \mathrm{~kg}$, e a idade média de $31,12 \pm 22,43$ meses. Não houve diferença estatística de peso dos animais entre os grupos $(\mathrm{P}=0,96)$. Em todas as avaliações do estudo, os resultados dos parâmetros de propriocepção (Tab. 2) e sensibilidade superficial e profunda (Tab. 3) foram idênticos nos membros pélvicos direito e esquerdo, evidenciando que a deposição dos tratamentos teve propagação bilateral simétrica.

No GS, não foi observado déficit motor (caracterizado por retorno à movimentação espontânea aos 7,50 \pm 4,18 minutos) ou sensitivo (retorno da sensibilidade profunda aos 5,83 $\pm 2,04$ minutos), e a recuperação da função motora dos membros pélvicos ocorreu logo após a recuperação da anestesia geral, aos 20 minutos da peridural, de acordo com o escore de atividade motora caracterizado por força motora preservada, marcha normal, com capacidade de ficar em pé e andar sem suporte. O resultado obtido nesse grupo mostra que a punção peridural lombossacra sem uso de fármacos anestésicos não causa alterações de caráter sensitivo ou motor.
No GL, o retorno à deambulação (Tab. 2) ocorreu em $57,50 \pm 17,54$ min e a recuperação da sensibilidade superficial, em 72,50 \pm 19,94 min. A literatura aponta 90 minutos de duração da lidocaína via peridural e, em média, 120 minutos para retorno do reflexo interdigital, corroborando os resultados do presente estudo (Gasparini et al., 2007; Valverde, 2008). A lidocaína foi utilizada nas comparações como controle positivo, pois as variáveis de latência, duração de bloqueio motor e sensitivo desse fármaco são bem elucidadas na literatura.

O bloqueio motor foi avaliado pelo tempo necessário para retorno à movimentação espontânea dos membros pélvicos, retorno à deambulação (Tab. 2) e escore de atividade motora ao longo do tempo (Tab. 3). Para retorno à movimentação espontânea, na comparação entre grupos, observou-se diferença entre os grupos GS $(7,50 \pm 4,18$ minutos $)$ e GR7,5 $(69,17 \pm 58,94$ minutos) $(\mathrm{P}=0,015)$, mas não entre $\operatorname{GL}(42,50 \pm$ 6,12 minutos) e GR7,5 $(69,17 \pm 58,94)$.

Apesar de não ter sido observada diferença entre os valores das médias de GL e GR7,5, clinicamente essa diferença é relevante. Assim, é possível que a ausência de diferença significativa entre esses dois grupos pode ser decorrente do número reduzido de animais por grupo (erro tipo II).

$\mathrm{Na}$ comparação do tempo de retorno à deambulação, GR7,5 (107,50 \pm 79,61 minutos) foi significativamente maior que GS $(9,17 \pm 3,76$ minutos) e GR5 (32,50 \pm 20,92 minutos $)$ $(\mathrm{P}=0,004)$ (Tab. 2), mostrando que a concentração de $7,5 \mathrm{mg} / \mathrm{mL}$ de ropivacaína, apesar de proporcionar um retorno rápido à movimentação espontânea, mantém reduzida a função muscular, o que acarretou prolongamento do tempo de retorno da capacidade de deambulação. Em dois 
estudos avaliando peridural com ropivacaína $7,5 \mathrm{mg} / \mathrm{mL}$, os autores observaram tempo mais prolongado para deambulação $(391,5 \pm 28,9$ minutos), contudo o volume administrado foi de 0,3 mL/kg (Albuquerque et al., 2010, 2013).

Tabela 2. Média e desvio-padrão, em minutos, do tempo para retorno da deambulação, da movimentação espontânea e da propriocepção em cães submetidos à anestesia peridural com $0,2 \mathrm{~mL} / \mathrm{kg}$ de solução $\mathrm{NaCl}$ 0,9\% (GS), lidocaína $2 \%$ sem vasoconstritor (GL), ropivacaína 5,0mg/mL sem vasoconstritor (GR5), ou ropivacaína $7,5 \mathrm{mg} / \mathrm{mL}$ sem vasoconstritor $(\mathrm{GR} 7,5)$

\begin{tabular}{|c|c|c|c|c|}
\hline \multirow{2}{*}{ Variáveis } & \multicolumn{4}{|c|}{ Grupos } \\
\hline & GS & GL & GR5 & GR7,5 \\
\hline Movimentação espontânea & $7,50 \pm 4,18^{(a)}$ & $42,50 \pm 6,12^{(\mathrm{ab})}$ & $26,67 \pm 21,37^{(\mathrm{ab})}$ & $69,17 \pm 58,94^{(\mathrm{b})}$ \\
\hline Deambulação & $9,17 \pm 3,76^{(\mathrm{a})}$ & $57,50 \pm 17,54^{(\mathrm{ab})}$ & $32,50 \pm 20,92^{(a)}$ & $107,50 \pm 79,61^{(b)}$ \\
\hline Propriocepção & $8,33 \pm 4,08^{(a)}$ & $85,00 \pm 50,79^{(\mathrm{b})}$ & $54,17 \pm 41,40^{(\mathrm{ab})}$ & $145,00 \pm 74,50^{(\mathrm{c})}$ \\
\hline
\end{tabular}

Letras minúsculas na linha representam diferença significativa de acordo com teste de Student-Newman-Keuls entre grupos $(\mathrm{P}<0,05)$.

Para o escore de atividade motora (Fig. 1), até o T10, não foi possível observar diferença entre os grupos. Como o período de latência dos anestésicos locais utilizados no presente estudo varia de 10 a 15 minutos (Valverde, 2008), os altos escores observados nesse período após a peridural, inclusive no GS, estão relacionados aos efeitos dos anestésicos gerais utilizados no presente estudo para garantir inconsciência e imobilidade durante a punção peridural. Os anestésicos gerais causam efeitos nos canais iônicos dependentes de voltagem no sistema nervoso; os agentes inalatórios possuem ação direta na transmissão neuromuscular na inibição dos canais para sódio da musculatura esquelética, fato que influencia a recuperação anestésica (Ginz et al., 2004). Na comparação entre grupos, GL se igualou ao GS apenas em T60, mas GR5 foi semelhante ao GS em todos os tempos, apontando novamente um menor grau de bloqueio motor dessa concentração de ropivacaína. Em GR7,5, o escore de deambulação se manteve diferente de GS até 180 minutos após a peridural, alcançando escore 1 somente em T300, o que sugere que ropivacaína a $7,5 \mathrm{mg} / \mathrm{mL}$, embora mais seletiva, mantém bloqueio motor parcial e ataxia por período superior à lidocaína.

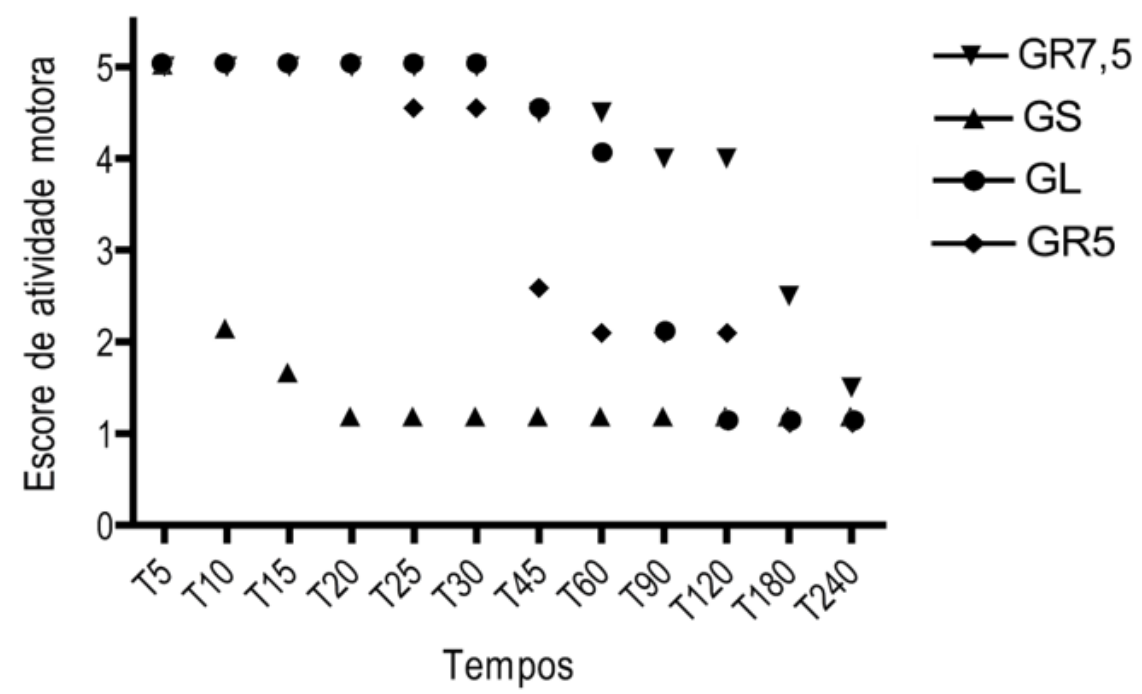

Figura 1. Mediana do escore de atividade motora (segundo Abelson et al., 2011), em relação ao tempo em minutos, de cães submetidos à anestesia peridural com $0,2 \mathrm{~mL} / \mathrm{kg}$ de solução $\mathrm{NaCl} 0,9 \%$ (GS), lidocaína $2 \%$ sem vasoconstritor (GL), ropivacaína $5,0 \mathrm{mg} / \mathrm{mL}$ sem vasoconstritor (GR5) ou ropivacaína 7,5mg/mL sem vasoconstritor $(\mathrm{GR} 7,5)$. 
O bloqueio sensitivo foi avaliado pela sensibilidade superficial e profunda (Tab. 3). O retorno da sensibilidade superficial foi mais precoce no GL (72,50 $\pm 19,94$ minutos) e no GR5 $(97,50 \pm 55,11$ minutos) em comparação ao GR7,5 (205,00 $\pm 129,27$ minutos) $(\mathrm{P}=0,009)$, mostrando que ropivacaína a $5 \mathrm{mg} / \mathrm{mL}$ oferece duração de bloqueio sensitivo semelhante à lidocaína. Tempo semelhante para retorno da sensibilidade superficial após peridural com ropivacaína $7,5 \mathrm{mg} / \mathrm{mL}$ foi relatado em outros estudos (Albuquerque et al., 2010; Andrade et al., 2019). Quanto ao retorno da sensibilidade profunda, este foi mais rápido no GR5 $(46,67 \pm$ $46,33$ minutos) e no GL (52,50 $\pm 20,68$ minutos $)$, sendo GR7,5 o grupo com período de retorno mais prolongado $(152,50 \pm 89,20$ minutos $)(\mathrm{P}=0,016)$, o que reforça que a ropivacaína a $5 \mathrm{mg} / \mathrm{mL}$ tem duração de ação semelhante à lidocaína.

Ao serem comparados os efeitos das diferentes concentrações de ropivacaína com a lidocaína, observou-se que o uso da ropivacaína a $5 \mathrm{mg} / \mathrm{mL}$ não promoveu incremento no retorno da movimentação espontânea e da sensibilidade profunda. Já a ropivacaína a $7,5 \mathrm{mg} / \mathrm{mL}$ promoveu incremento de $42 \%$ no efeito de bloqueio motor, e de $66 \%$ no efeito sensitivo, quando comparada à lidocaína. Esses resultados reforçam que o uso de ropivacaína em baixas concentrações possui efeitos similares à lidocaína e que ropivacaína a $7,5 \%$, apesar de apresentar bloqueio motor seletivo, incrementa o retorno da função muscular.

Tabela 3. Média e desvio-padrão, em minutos, do tempo para retorno da sensibilidade superficial e profunda em cães submetidos à anestesia peridural com $0,2 \mathrm{~mL} / \mathrm{kg}$ de solução $\mathrm{NaCl} 0,9 \%$ (GS), lidocaína $2 \%$ sem vasoconstritor $(\mathrm{GL})$, ropivacaína $5,0 \mathrm{mg} / \mathrm{mL}$ sem vasoconstritor (GR5), ou ropivacaína 7,5mg/mL sem vasoconstritor $(\mathrm{GR} 7,5)$

\begin{tabular}{lcccc}
\multicolumn{1}{c}{ Variáveis } & \multicolumn{1}{c}{$\begin{array}{c}\text { Grupos } \\
\text { GS }\end{array}$} & GL & GR5 & GR7,5 \\
\hline $\begin{array}{l}\text { Sensibilidade } \\
\text { superficial }\end{array}$ & $7,50 \pm 2,74^{\text {(a) }}$ & $72,50 \pm 19,94^{\text {(a) }}$ & $97,50 \pm 55,11^{\text {(a) }}$ & $205,00 \pm 129,27^{\text {(b) }}$ \\
$\begin{array}{l}\text { Sensibilidade } \\
\text { profunda }\end{array}$ & $5,83 \pm 2,04^{(\text {a) }}$ & $52,50 \pm 20,68^{(\text {a) }}$ & $46,67 \pm 46,33^{\text {(a) }}$ & $152,50 \pm 89,20^{\text {(b) }}$ \\
Lefrand & & &
\end{tabular}

Letras minúsculas na linha representam diferença significativa de acordo com teste de Student-Newman-Keuls entre grupos $(\mathrm{P}<0,05)$.

A padronização do posicionamento dos pacientes em esfinge, com os membros pélvicos tracionados cranialmente, foi realizada para elevar a acurácia da técnica pelo aumento do espaço entre L7 e S1 (Concetto et al., 2012). Para a confirmação do correto posicionamento da agulha durante a punção peridural, a literatura aponta que tanto a verificação pelo teste da gota pendente quanto a verificação por meio da ausência de deformidade da bolha no anestésico de forma isolada possuem baixa acurácia (Adami e Gendron, 2017). Desse modo, no presente estudo, empregaram-se as duas técnicas em conjunto e a injeção só foi realizada após sucção da gota e ausência de deformação da bolha de ar na seringa.

Considerando que a velocidade da deposição de fármacos pela via peridural está diretamente relacionada com o aumento da pressão no espaço peridural e consequentemente com os efeitos adversos decorrentes da anestesia peridural (Son et al., 2014), no presente estudo optou-se pela padronização do tempo de administração do fármaco em dois minutos. Apesar dos riscos de depressão cardiovascular e hipoventilação (Bosmans et al., 2009; Zaki, 2009; Bosmans et al., 2011; Dias et al., 2018), no presente estudo não foram observadas reações adversas de caráter sistêmico ou local em nenhum dos cães. Isso pode ser resultado do volume controlado, da velocidade de administração e do fato de, no presente estudo, os animais não estarem sob influência de agentes anestésicos gerais durante as avaliações.

O tempo de bloqueio motor e sensitivo da ropivacaína por via peridural é dose-dependente (Feldman et al., 1996). Sabe-se que a variação na sua concentração não altera o período de latência, no entanto afeta a duração do bloqueio motor e sua extensão. Em estudo comparando duas doses e duas concentrações de ropivacaína, a duração do bloqueio em níveis mais craniais foi mais curta nos grupos que receberam ropivacaína a $5 \mathrm{mg} / \mathrm{mL}$ comparada a grupos que receberam ropivacaína $7,5 \mathrm{mg} / \mathrm{mL}$ (Duke et al., 2000). A administração peridural de $0,2 \mathrm{~mL} / \mathrm{kg}$ de ropivacaína a 
$2,5 \mathrm{mg} / \mathrm{mL}$ e $5 \mathrm{mg} / \mathrm{mL}$ não promoveu analgesia satisfatória em cães submetidos a cirurgias ortopédicas invasivas, tendo a concentração mais alta acarretado bloqueio motor mais prolongado (Adami et al., 2012), como pode ser observado também no presente estudo.

Em outro estudo, a administração de ropivacaína $7,5 \mathrm{mg} / \mathrm{mL}$ resultou em retorno da sensibilidade superficial similar ao presente trabalho $(254,0 \pm$ 30,4 minutos) e sua associação com tramadol causou aumento no período de latência, enquanto a associação à fentanila prolongou o efeito de anestesia $(338,5 \pm 33,8$ minutos para retorno à posição quadrupedal) (Silva et al., 2008), sugerindo que, possivelmente, a associação de ropivacaína a $5 \mathrm{mg} / \mathrm{ml}$ com opioide tenha maior efeito de bloqueio sensitivo que seu uso isolado.

\section{CONCLUSÕES}

A ropivacaína $0,5 \%$ promove bloqueio motor e sensitivo semelhante à lidocaína 2\%. Para ropivacaína $0,75 \%$, apesar do retorno precoce da movimentação espontânea, sua administração peridural está relacionada a maior tempo de recuperação da função muscular e da coordenação motora, caracterizado por maior tempo para retorno à deambulação e bloqueio sensitivo mais prolongado que lidocaína e ropivacaína $0,5 \%$.

\section{REFERÊNCIAS}

ABELSON, A.L.; ARMITAGE-CHAN, E.; LINDSEY, J.C.; WETMORE, L.A. A comparison of epidural morphine with low dose bupivacaine versus epidural morfphine alone on motor and respiratory function in dogs following splenectomy. Vet. Anaesth. Analg., v.38, p.213223, 2011.

ADAMI, C.; GENDRON, K. What is the evidence? The issue of verifying correct needle position during epidural anaesthesia in dogs. Vet. Anaesth. Analg., v.44, p.212-218, 2017.

ADAMI, C.; VERES-NYÉKI, K.; SPADAVECCHIA, C. et al. Evaluation of perioperative epidural analgesia with ropivacaine, ropivacaine and sufentanil, and ropivacaine, sufentanil and epinephrine in isoflurane anesthetized dogs undergoing tibial plateau levelling osteotomy. Vet. J., v.194, p.229-234, 2012.
ALBUQUERQUE, V.B.; SOUZA, T F.B.; VIVAN, M.C.R. et al. Influência do butorfanol sobre os períodos de latência e de ação da ropivacaína pela via peridural na ovariossalpingohisterectomia em cadela. Arq. Bras. Med. Vet. Zootec., v.62, p.806-811, 2010.

ALBUQUERQUE, V.B.; SOUZA, T.F.B.; VIVAN, M.C.R. et al. Ropivacaína isolada ou associada à morfina, butorfanol ou tramadol pela via peridural em cadelas para realização de ovariosalpingohisterectomia. Vet. Zootec., v.20, p.111-123, 2013.

ALMEIDA, R.M.; ESCOBAR, A.; MAGUILNIK, S. Comparison of analgesia provided by lidocaine, lidocaine-morphine or lidocaine-tramadol delivered epidurally in dogs following orchiectomy. Vet. Anaesth. Analg., v.37, p.542-549, 2010.

ANDRADE, N.R.; SANTOS, B.C.P.; CAIRES, L.P. et al. Ropivacaína isolada ou associada à metadona ou à morfina, pela via epidural, em cadelas submetidas à ovário-histerectomia. Arq. Bras. Med. Vet. Zootec., v.71, p.430-438, 2019.

BOSMANS, T.; SCHAUVLIEGE, S.; GASTHUYS, F. et al. Cardiovascular effects of epidural administration of methadone, ropivacaine $0.75 \%$ and their combination in isoflurane anaesthetized dogs. Vet. Anaesth. Analg., v.38, p.146-157, 2011.

BOSMANS, T.; SCHAUVLIEGE, S.; GASTHUYS, F. et al. Transient unilateral Horner's syndrome after epidural ropivacaine in a dog. Vet. Anaesth. Analg., v.36, p.401-406, 2009.

CAMPOY, L.; READ, M.; PERALTA, S. Técnicas de anestesia local e analgesia em cães e gatos. In: TRANQUILI, W.J.; THURMON, J.C.; GRIMM, K.A. Lumb \& Jones veterinary anesthesia and analgesia. 5.ed. Rio de Janeiro: Roca, 2015. p.2413-2503.

CONCETTO, S.D.; MANDSAGER, R.E.; RIEBOLD, T.W. et al. Effect of hind limb position on the craniocaudal length of the lumbosacral space in anesthetized dogs. Vet. Anaesth. Analg., v.39, p.99-105, 2012.

DIAS, R.S.G.; SOARES, J.H.N.; CASTRO, D.S. et al. Cardiovascular and respiratory effects of lumbosacral epidural bupivacaine in isofluraneanesthetized dogs: the effects of two volumes of $0.25 \%$ solution. Plos One., v.13, p.1$16,2018$. 
DUKE, T.; CAULKETT, N.A.; BALL, S.D.; REMEDIOS, AM. Comparative analgesic and cardiopulmonary effects of bupivacaine and ropivacaine in the epidural space of the conscious dog. Vet. Anaesth. Analg., v.27, p.13-21, 2000.

FELDMAN, H.S.; DVOSKIN, S.; ARTHUR, G.R.; DOUCETTE, A.M. Antinociceptive and motor-blocking efficacy of ropivacaine and bupivacaine after epidural administration in the dog. Reg. Anesth., v.21, p.318-326, 1996.

GARCIA-PEREIRA, F. Epidural anesthesia and analgesia in small animal practice: an update. Vet. J., v.242, p.24-32, 2018.

GASPARINI, S.S.; LUNA, S.P.L.; CASSU, R.N. et al. Anestesia epidural com ropivacaína, lidocaína ou associação de lidocaína e xilazina em cães. Efeitos cardiorrespiratório e analgésico. Cienc. Rural, v.37, p.418-424, 2007.

GINZ, H.F.; ZORZATO, F.; IAIAZZO, P.A.; URWYLER, A. Effect of three anaesthetic techniques on isometric skeletal muscle strength. Br. J. Anaesth., v.92, p.367-372, 2004.

JONES, R.S. Epidural analgesia in the dog and cat. Vet. J., v.161, p.123-131, 2001.

MARTIN-FLORES, M. Clinical pharmacology and toxicologyof local anesthetics and adjuncts. In: CAMPOY, L.; READ, M.R Small animal regional anesthesia and analgesia. Iowa: Blackwell Scientific Pub., 2013. p.25-41.
OTERO, P.E.; CAMPOY, L. Epidural and spinal anesthesia. In: CAMPOY, L.; READ, M.R. Small animal regional anesthesia and analgesia. Iowa: Blackwell Scientific Pub., 2013. p.227-259.

SILVA, B.M.; MATSUBARA, L.M.; ALBUQUERQUE, V.B. et al. Ropivacaína isolada e associada ao fentanil ou ao tramadol administrados pela via peridural em cães. Cienc. Rural, v.38, p.2197-2202, 2008.

SON, W.G.; JANG, M.; LEE, L.Y. et al. The effect of epidural injection speed on epidural pressure and distribution of solution in anesthetized dogs. Vet. Anaesth. Analg., v.41, p.526-533, 2014.

STEAGALL, P.V.M.; SIMON, B.T.; NETO, F.J.T.; LUNA, S.P.L. An update on drugs used for lumbosacral epidural anesthesia and analgesia in dogs. Front. Vet. Sci., v.4, p.1-12, 2017.

VALVERDE, A. Epidural analgesia and anesthesia in dogs and cats. Vet. Clin. Small Anim., v.38, p.1205-1230, 2008.

ZAKI, S. Local Analgesia Using Nerve Block Techniques. In: ZAKI, S.; MUSK, G. anaesthesia and analgesia. Sydney: Centre for Veterinary Education, 2009. p.33-42. 